\title{
ON THE LOCATION OF ROOTS OF STEINER POLYNOMIALS
}

\author{
MARTIN HENK AND MARÍA A. HERNÁNDEZ CIFRE
}

\begin{abstract}
We investigate the roots of relative Steiner polynomials of convex bodies. In dimension 3 we give a precise description of their location in the complex plane and we study the analogous problem in higher dimensions. In particular, we show that the roots (in the upper half plane) form a convex cone; for dimensions $\leq 9$ this cone is completely contained in the (open) left half plane, which is not true in dimensions $\geq 12$. Moreover, we characterize certain special families of convex bodies by means of properties of their roots.
\end{abstract}

\section{INTRODUCTION}

Let $\mathcal{K}^{n}$ be the set of all convex bodies, i.e., compact convex sets, in the $n$ dimensional Euclidean space $\mathbb{R}^{n}$, and let $B_{n}$ be the $n$-dimensional unit ball. The subset of $\mathcal{K}^{n}$ consisting of all convex bodies with non-empty interior is denoted by $\mathcal{K}_{0}^{n}$. The volume of a set $M \subset \mathbb{R}^{n}$, i.e., its $n$-dimensional Lebesgue measure, is denoted by $\operatorname{vol}(M)$. For two convex bodies $K, E \in \mathcal{K}^{n}$ and a non-negative real number $\lambda$, the volume of the so called relative outer parallel body of $K$ with respect to $E, K+\lambda E$, is expressed as a polynomial of degree $n$ in $\lambda$ and it can be written as

$$
\operatorname{vol}(K+\lambda E)=\sum_{i=0}^{n}\left(\begin{array}{l}
n \\
i
\end{array}\right) \mathrm{W}_{i}(K ; E) \lambda^{i} .
$$

This expression is called Minkowski-Steiner formula or relative Steiner formula of $K$. The coefficients $\mathrm{W}_{i}(K ; E)$ are the relative quermassintegrals of $K$, and they are a special case of the more general defined mixed volumes for which we refer to [19, s. 5.1]. In particular, we have $\mathrm{W}_{0}(K ; E)=\operatorname{vol}(K)$, $\mathrm{W}_{n}(K ; E)=\operatorname{vol}(E)$ and $\mathrm{W}_{i}(K ; E)=\mathrm{W}_{n-i}(E ; K)$. If $E=B_{n}$, (1.1) becomes the classical Steiner formula [20], and $\mathrm{W}_{i}\left(K ; B_{n}\right)$, for short denoted by $\mathrm{W}_{i}(K)$, is the classical $i$-th quermassintegral of $K$.

2000 Mathematics Subject Classification. Primary 52A20, 52A39; Secondary 30C15.

Key words and phrases. Roots of Steiner polynomial, tangential bodies, constant width sets, sausages.

Second author is supported in part by Subdirección General de Proyectos de Investigación (MCI) MTM2009-10418 and by "Programa de Ayudas a Grupos de Excelencia de la Región de Murcia", Fundación Séneca, 04540/GERM/06. 
In the following, $E \in \mathcal{K}_{0}^{n}$ will be always a fixed convex body with nonempty interior, and for $K \in \mathcal{K}^{n}$ we will write

$$
f_{K ; E}(z)=\sum_{i=0}^{n}\left(\begin{array}{c}
n \\
i
\end{array}\right) \mathrm{W}_{i}(K ; E) z^{i}
$$

to denote the Steiner polynomial of $K$ relative to $E$, regarded as a formal polynomial in a complex variable $z \in \mathbb{C}$. We are interested in the location of the roots of $f_{K ; E}(z)$. To this end let $\mathbb{C}^{+}=\{z \in \mathbb{C}: \operatorname{Im}(z) \geq 0\}$ be the set of complex numbers with non-negative imaginary part, and let

$$
\mathcal{R}(n, E)=\left\{z \in \mathbb{C}^{+}: f_{K ; E}(z)=0 \text { for some } K \in \mathcal{K}^{n}\right\}
$$

be the set of all roots of $f_{K ; E}(z), K \in \mathcal{K}^{n}$, in the upper half plane.

Theorem 1.1. $\mathcal{R}(n, E)$ is a convex cone containing the non-positive real axis.

By the isoperimetric inequality (cf. e.g., [19, p. 318]) it is easy to see that in the plane, $\mathcal{R}(2, E)$ is exactly the non-positive real axis. In order to describe the 3-dimensional cone we need the so called cap-bodies. A convex body $K \in \mathcal{K}^{n}$ is called a cap-body of $L \in \mathcal{K}^{n}$ if $K$ is the convex hull of $L$ and countably many points such that the line segment joining any pair of these points intersects $L$. With this notation we can precisely describe $\mathcal{R}(3, E)$ (see Figure 1).

Theorem 1.2. If $E \in \mathcal{K}_{0}^{3}$ is a cap-body of a planar convex body then

$$
\mathcal{R}(3, E)=\left\{x+y \mathrm{i} \in \mathbb{C}^{+}: x+\sqrt{3} y \leq 0\right\},
$$

otherwise

$$
\mathcal{R}(3, E)=\left\{x+y \mathrm{i} \in \mathbb{C}^{+}: x+\sqrt{3} y<0\right\} \cup\{0\} .
$$

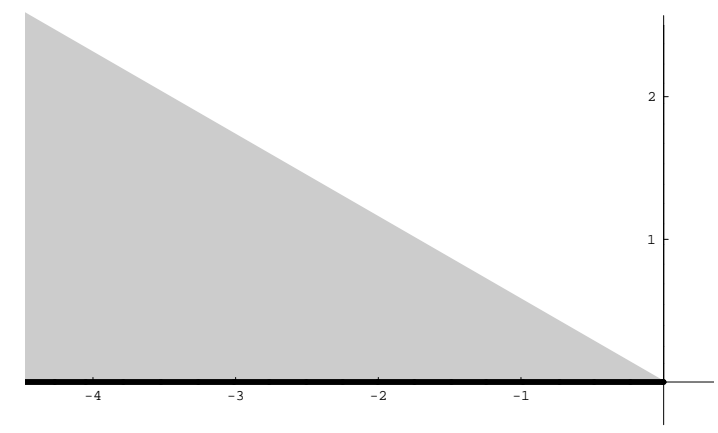

FiguRE 1. Roots of 3-dimensional Steiner polynomials in the upper half plane.

In particular, we note that the set $\mathcal{R}(2, E)$, as well as the closure of $\mathcal{R}(3, E)$, are independent of the gauge body $E$. It seems to be quite likely 
that this holds true in any dimension. Our result is restricted to dimension 3 since in higher dimension we do not have enough information about the so called "complete system" of inequalities among the quermassintegrals (cf. e.g., [6, Problem 6.1]). We also do not know whether in general $\mathcal{R}(n, E) \subseteq \mathcal{R}(n+1, E)$ or whether for particular gauge bodies (e.g. $E=B_{n}$ ) the cone is always half open as in the case $n=3$.

The property that all the roots of 3-dimensional Steiner polynomials lie in the left half plane was part of a conjecture posed by Sangwine-Yager [17] (cf. e.g., [18, p. 65]) which was motivated by a problem of Teissier [21. There it was in particular claimed that Steiner polynomials are "weakly" stable polynomials, i.e.,

$$
\mathcal{R}(n, E) \subseteq\left\{z \in \mathbb{C}^{+}: \operatorname{Re}(z) \leq 0\right\} .
$$

This inclusion is known to be true for dimensions $\leq 5$ (cf. e.g., [21]), but in 7 it was shown to be false in dimensions $\geq 12$ for a special family of 3 tangential bodies (see also 13 for another family of high dimensional convex bodies with this property). Here we narrow the gap between these values of the dimension by showing the following result.

\section{Proposition 1.1.}

i) For $n \leq 9$ we have $\mathcal{R}(n, E) \subseteq\left\{z \in \mathbb{C}^{+}: \operatorname{Re}(z)<0\right\} \cup\{0\}$.

ii) For $n \geq 12$ we have $\left\{z \in \mathbb{C}^{+}: \operatorname{Re}(z) \leq 0\right\} \subset \mathcal{R}(n, E)$.

For further information on the roots of Steiner polynomials in the context of Teissier's problem we refer to [7, 8, 10, 11, 13].

Next we consider the problem to characterize convex bodies by properties of the roots of their Steiner polynomials. To this end we denote by

$$
\mathrm{r}(K ; E)=\max \left\{r \geq 0: \exists x \in \mathbb{R}^{n} \text { with } x+r E \subseteq K\right\}
$$

the inradius of $K \in \mathcal{K}^{n}$ with respect to $E$. If $E=B_{n}$ then $\mathrm{r}\left(K ; B_{n}\right)$, for short $\mathrm{r}(K)$, is the classical inradius, i.e., the radius of a largest Euclidean ball contained in $K$. It is easy to check that for an $l$-dimensional convex body $L \in \mathcal{K}^{n}$, the Steiner polynomial of $K=L+\mathrm{r} B_{n}$ has the $(n-l)$-fold root $-\mathrm{r}=-\mathrm{r}(K)$. We believe that those bodies are completely characterized by this property.

Conjecture 1.1. Let $K \in \mathcal{K}^{n}$ and let $l \in\{0, \ldots, n-1\}$. Then $-\mathrm{r}(K)$ is an $(n-l)$-fold root of $f_{K ; B_{n}}(z)$ if and only if $K=L+\mathrm{r}(K) B_{n}$ for some $L \in \mathcal{K}^{n}$ with $\operatorname{dim} L=l$.

The case $l=0$ is known to be true (see [9]) and will also follow from a more general statement in Section 3 (Proposition 3.2). The case $l=n-1$ is related to a conjecture of Matheron [15] (see also [19, p. 212]) claiming that $B_{n}$ is a summand of $K \in \mathcal{K}^{n}$, i.e., $K=L+B_{n}$ for some $L \in \mathcal{K}^{n}$, if and only if the volume of the inner parallel body $K \sim \lambda B_{n}=\left\{x \in K: x+\lambda B_{n} \subseteq K\right\}$ is given by the so called alternating Steiner polynomial, i.e.,

$$
\operatorname{vol}\left(K \sim \lambda B_{n}\right)=f_{K ; B_{n}}(-\lambda), \lambda \in(0,1) .
$$


In fact, this was conjectured by Matheron for any gauge body $E \in \mathcal{K}_{0}^{n}$, not only for $B_{n}$. Observe that for $K \in \mathcal{K}^{n}$ with $\mathrm{r}(K)=1$, (1.3) implies that $-\mathrm{r}(K)$ is a root of $f_{K ; B_{n}}(z)$. So far Matheron's conjecture is only known to be true in the case $n=2$ (see [15]). Based on the equality case in Bonnesen's inequality, namely,

$$
\mathrm{W}_{0}(K ; E)-2 \mathrm{~W}_{1}(K ; E) \mathrm{r}(K ; E)+\mathrm{W}_{2}(K ; E) \mathrm{r}(K ; E)^{2} \leq 0
$$

with equality if and only if $K=L+\mathrm{r}(K ; E) E$ for $\operatorname{dim} L \leq 1$ (see [1, pp. 3336], [3]), we also see that Conjecture 1.1 is true for any gauge body $E$ in dimension 2. Regarding the "sausage" case $l=1$ of the conjecture above we are able to prove the following weakening.

Theorem 1.3. Let $K \in \mathcal{K}^{n}, n \geq 2$. Then $-\mathrm{r}(K)$ is an $(n-1)$-fold root of $f_{K ; B_{n}}(z)$ and all its 2 -dimensional projections onto any 2 -dimensional linear subspace have inradius $\mathrm{r}(K)$ if and only $K$ is a sausage with inradius $\mathrm{r}(K)$, i.e., there exists a line segment $L$ such that $K=L+\mathrm{r}(K) B_{n}$.

We note that, for instance, cap-bodies of $B_{n}$ (see Section 3 for the definition) have the property that all their 2-dimensional projections have the same inradius as the body, namely, $1=\mathrm{r}\left(B_{n}\right)$. In the last section we show that cap-bodies, as well as the more general class of $p$-tangential bodies, can be characterized via the roots of their Steiner polynomials (see Proposition 3.1 and Corollary 3.1). Moreover, we investigate the roots of Steiner polynomials of constant width sets and obtain the following result.

Proposition 1.2. The roots of Steiner polynomials $f_{K ; B_{n}}(z)$ of convex bodies $K$ of constant width $\mathrm{b}$ are symmetric with respect to $-\mathrm{b} / 2$, i.e., $f_{K ; B_{n}}(z)=0$ if and only if $f_{K ; B_{n}}(-z-\mathrm{b})=0$.

The paper is organized as follows. At the beginning of Section 2 we give some preliminary results on properties of roots of Steiner polynomials, which are then needed for the proofs of Theorems 1.1 and 1.2 and Proposition 1.1 . In Section 3 we provide the announced characterization of cap-bodies and $p$ tangential bodies and we present the proofs of Theorem 1.3 and Proposition 1.2 both results will be consequences of more general statements.

\section{On the LOCATion of the Roots of Steiner POLynomials}

First we collect some properties on the behavior of the roots of Steiner polynomials when we change a bit the involved bodies.

Lemma 2.1. Let $\gamma$ be a root of the Steiner polynomial $f_{K ; E}(z)$.

i) Let $\lambda>0$. Then $\lambda \gamma$ is a root of $f_{\lambda K ; E}(z)$.

ii) Let $a \geq 0$. Then $\gamma-a$ is a root of $f_{K+a E ; E}(z)$.

iii) Let $\gamma=x+y \mathrm{i}$ with $x<0$, and let $0<\rho \leq 1$. Then $x+(\rho y) \mathrm{i}$ is a root of $f_{\rho K+x(\rho-1) E ; E}(z)$. 
Proof. By the homogeneity of the quermassintegrals we have $\mathrm{W}_{i}(\lambda K ; E)=$ $\lambda^{n-i} \mathrm{~W}_{i}(K ; E)$ for $i=0, \ldots, n$, and hence $f_{\lambda K ; E}(z)=\lambda^{n} f_{K ; E}(z / \lambda)$, which shows i). Since for any $a, \lambda \geq 0$

$$
\operatorname{vol}(K+a E+\lambda E)=\operatorname{vol}(K+(a+\lambda) E)=\sum_{i=0}^{n}\left(\begin{array}{c}
n \\
i
\end{array}\right) \mathrm{W}_{i}(K ; E)(a+\lambda)^{i},
$$

we have $f_{K+a E ; E}(z)=f_{K ; E}(a+z)$, which implies ii). Finally, iii) is just a combination of ii) and i), since $\rho K+x(\rho-1) E=\rho(K+[x(\rho-1) / \rho] E)$.

Next we give the proof of Theorem 1.1

Proof of Theorem 1.1. First we note that -1 is a root of the polynomial $f_{E ; E}(z)$ since in this case $\mathrm{W}_{i}(E ; E)=\mathrm{W}_{0}(E ; E)$ for all $i=1, \ldots, n$, and thus $f_{E ; E}(z)=\mathrm{W}_{0}(E ; E)(z+1)^{n}$. Hence, by Lemma 2.1 i) the negative real axis is contained in $\mathcal{R}(n, E)$. Next observe that also 0 is a root of $f_{K ; E}(z)$ for any $K \in \mathcal{K}^{n}$ with $\operatorname{dim} K<n$, because in this case $\mathrm{W}_{0}(K ; E)=0$. Together with Lemma 2.1 i) this shows that $\mathcal{R}(n, E)$ is a rayset, i.e., for $\gamma \in \mathcal{R}(n, E)$ and any $\lambda \geq 0$ we also have $\lambda \gamma \in \mathcal{R}(n, E)$. Hence, with Lemma 2.1 ii) this shows that $\mathcal{R}(n, E)$ is a convex cone. In fact, given roots $\gamma_{i}=x_{i}+y_{i} \mathrm{i} \in \mathcal{R}(n, E)$ of polynomials $f_{K_{i} ; E}(z), i=1,2$, and $\rho \in(0,1)$, we can construct a convex body $M$ such that $\rho \gamma_{1}+(1-\rho) \gamma_{2}$ is a root of $f_{M ; E}(z)$ as follows. Since $\mathcal{R}(n, E)$ contains the non-positive real numbers but no positive numbers, we may assume that not both roots are real numbers. Let $x_{1} / y_{1}=\max \left\{x_{i} / y_{i}: y_{i}>0, i=1,2\right\}$ and let $\mu=y_{1} /\left(\rho y_{1}+(1-\rho) y_{2}\right)$. By the choice of $x_{1} / y_{1}$ we have

$$
\nu=\mu\left(\rho x_{1}+(1-\rho) x_{2}\right)=y_{1} \frac{\rho x_{1}+(1-\rho) x_{2}}{\rho y_{1}+(1-\rho) y_{2}} \leq x_{1},
$$

since it is easy to see that the above function is increasing in $\rho \in(0,1)$. So we know by Lemma 2.1 ii) that $\nu+y_{1}$ i is a root of $f_{K_{1}+\left(x_{1}-\nu\right) E ; E}$ and thus, by Lemma 2.1 i) $\rho \gamma_{1}+(1-\rho) \gamma_{2}$ is a root of the Steiner polynomial of $M=(1 / \mu)\left(K_{1}+\left(x_{1}-\nu\right) E\right)$.

In [7, Theorem 1.2, Remark 3.2] it was shown that for dimensions $n \geq 12$ there exist convex bodies $K$ such that some of the roots of $f_{K ; B_{n}}(z)$ have positive real part. The constructions of these so called 3 -tangential bodies of $B_{n}$ can be easily generalized to an arbitrary gauge body $E \in \mathcal{K}_{0}^{n}$. Hence we know by Theorem 1.1 that, for $n \geq 12$,

$$
\left\{z \in \mathbb{C}^{+}: \operatorname{Re}(z) \leq 0\right\} \subset \mathcal{R}(n, E),
$$

which is the second statement in Proposition 1.1 .

The first statement of this proposition describes a stability property of Steiner polynomials. We recall that a real polynomial is said to be stable if all its roots have (strict) negative real part. Hence, again on account of Theorem 1.1. Proposition 1.1 i) says that for dimension $n \leq 9$ Steiner 
polynomials are stable if we neglect for a moment the root 0 . The main ingredient in order to prove it are the inequalities

$$
\begin{aligned}
& \text { (2.1) } \mathrm{W}_{i}(K ; E)^{2} \geq \mathrm{W}_{i-1}(K ; E) \mathrm{W}_{i+1}(K ; E), \quad 1 \leq i \leq n-1, \\
& (2.2) \quad \mathrm{W}_{i}(K ; E) \mathrm{W}_{j}(K ; E) \geq \mathrm{W}_{k}(K ; E) \mathrm{W}_{l}(K ; E), \quad 0 \leq k<i<j<l \leq n, \\
& \text { particular cases of the Aleksandrov-Fenchel inequality (see e.g. [19, s. 6.3]). }
\end{aligned}
$$

Proof of Proposition 1.1. It remains to show part i). We know 0 is a root of $f_{L ; E}(z)$ if and only if $\operatorname{dim} L=l<n$, and in this case we may write $f_{L ; E}(z)=$ $z^{n-l} \sum_{i=n-l}^{n}\left(\begin{array}{c}n \\ i\end{array}\right) \mathrm{W}_{i}(L ; E) z^{i-n+l}$ with $\mathrm{W}_{i}(L ; E)>0$ for $n-l \leq i \leq n$. Hence we have to show that all polynomials of the type $\sum_{i=n-l}^{n}\left(\begin{array}{c}n \\ i\end{array}\right) \mathrm{W}_{i}(L ; E) z^{i-n+l}$, $\mathrm{W}_{i}(L ; E)>0$ for $n-l \leq i \leq n, 0<l \leq n \leq 9$, are stable.

For $n \leq 5$ it was already pointed out by Teissier [21, p. 103] that inequalities (2.1) and (2.2) guarantee that the Routh-Hurwitz criterion for stability of polynomials (see e.g. [14, p. 181]) is fulfilled, and so it remains to consider the case $n \geq 6$.

Here we use the following stability criterion [16, Theorem 3] (see also an independent proof in [12, Theorem 1]). A real polynomial $f(z)=\sum_{i=0}^{n} a_{i} z^{i}$, with $a_{i}>0$ for $i=0, \ldots, n$, is stable if $a_{i-1} a_{i+2} \leq \beta a_{i} a_{i+1}, i=1, \ldots, n-2$, where $\beta \approx 0.4655$ is the only real solution of $z(z+1)^{2}=1$. Again it is easy to check that (2.1) and (2.2) imply this criterion.

So only in dimensions $n=10,11$ we do not know whether Steiner polynomials can have roots with positive real parts. Obviously, by the convexity of the set $\mathcal{R}(n, E)$ the existence of a root with positive real part also implies the existence of a pure imaginary complex root. However, not all roots can be of that type. More precisely

Proposition 2.1. There exists no convex body $K \in \mathcal{K}^{n}$ such that all roots of $f_{K ; E}(z)$ are imaginary pure complex numbers (excluding the real root always existing in odd dimension).

Proof. Notice first that if $\operatorname{dim} K \leq 1$ then the Steiner polynomial is of degree $\leq 1$ and there is nothing to prove. Thus we can assume that $\operatorname{dim} K \geq 2$.

For $n$ even, let $K \in \mathcal{K}^{n}$ be a convex body such that all roots of $f_{K ; E}(z)$ are $\left\{ \pm y_{j} \mathrm{i}, j=1, \ldots, n / 2\right\}$, with $y_{j} \in \mathbb{R}_{>0}$. Then we get

$$
f_{K ; E}(z)=\mathrm{W}_{n}(K ; E) \prod_{j=1}^{n / 2}\left(z^{2}+y_{j}^{2}\right),
$$

which implies $\mathrm{W}_{2 i+1}(K ; E)=0$ for all $i=0, \ldots,(n-2) / 2$. In particular, $\mathrm{W}_{n-1}(K ; E)=0$ and hence $\operatorname{dim} K=0$, which contradicts our assumption.

For $n$ odd, let $K \in \mathcal{K}^{n}$ be a convex body such that the roots of $f_{K ; E}(z)$ are $\left\{-x, \pm y_{j} \mathrm{i}, j=1, \ldots,(n-1) / 2\right\}$, with $x \geq 0$ and $y_{j} \in \mathbb{R}_{>0}$. From

$$
f_{K ; E}(z)=\mathrm{W}_{n}(K ; E)(z-x) \prod_{j=1}^{(n-1) / 2}\left(z^{2}+y_{j}^{2}\right)
$$


we get

$$
\begin{aligned}
\sum_{j=1}^{(n-1) / 2} y_{j}^{2} & =\left(\begin{array}{l}
n \\
2
\end{array}\right) \frac{\mathrm{W}_{n-2}(K ; E)}{\mathrm{W}_{n}(K ; E)}, \quad x \sum_{j=1}^{(n-1) / 2} y_{j}^{2}=\left(\begin{array}{c}
n \\
3
\end{array}\right) \frac{\mathrm{W}_{n-3}(K ; E)}{\mathrm{W}_{n}(K ; E)} \\
x & =n \frac{\mathrm{W}_{n-1}(K ; E)}{\mathrm{W}_{n}(K ; E)} .
\end{aligned}
$$

Thus we obtain the relation

$$
\frac{3 n}{n-2} \mathrm{~W}_{n-2}(K ; E) \mathrm{W}_{n-1}(K ; E)=\mathrm{W}_{n}(K ; E) \mathrm{W}_{n-3}(K ; E) .
$$

For $\operatorname{dim} K \geq 2$ the left hand side in the above equality is positive, and since it holds $\mathrm{W}_{n-2}(K ; E) \mathrm{W}_{n-1}(K ; E) \geq \mathrm{W}_{n}(K ; E) \mathrm{W}_{n-3}(K ; E)$ for all convex bodies (cf. (2.2)), we get the desired contradiction.

In Section 3 we will show a kind of generalization of the proposition above, proving that all roots of $f_{K ; E}(z)$ cannot lie on two symmetric parallel lines of the form $\{x+y \mathrm{i}: x= \pm a\}$, except for the case of an $n$-fold real root (Proposition 3.2).

Next we come to the proof of Theorem 1.2 in which we give a description of the cone $\mathcal{R}(3, E)$ (see Figure 1 ).

Proof of Theorem 1.2. Let $-a+b \mathrm{i} \in \mathbb{C}^{+}$be a root of a Steiner polynomial $f_{K ; E}(z)$ for some $K \in \mathcal{K}^{3}$ and $E \in \mathcal{K}_{0}^{3}$. By Proposition 1.1 we may assume that both $a, b>0$ and we have to show that $\sqrt{3} b \leq a$. Moreover, let $-c$, $c \geq 0$, be the real root of $f_{K ; E}(z)$. Then we have the identities

$2 a+c=3 \frac{\mathrm{W}_{2}(K ; E)}{\mathrm{W}_{3}(K ; E)}, \quad a^{2}+b^{2}+2 a c=3 \frac{\mathrm{W}_{1}(K ; E)}{\mathrm{W}_{3}(K ; E)}, \quad c\left(a^{2}+b^{2}\right)=\frac{\mathrm{W}_{0}(K ; E)}{\mathrm{W}_{3}(K ; E)}$.

Inequalities (2.1) for $i=2$ and $i=1$ yield in terms of $a, b, c$, respectively,

$$
\begin{gathered}
(a-c)^{2} \geq 3 b^{2}, \\
\left(a^{2}-3 b^{2}\right) c^{2}-2 a\left(a^{2}+b^{2}\right) c+\left(a^{2}+b^{2}\right)^{2} \geq 0 .
\end{gathered}
$$

The first one is equivalent to $c \leq a-\sqrt{3} b$ or $c \geq a+\sqrt{3} b$. In the first case we are done and so we are left with the case $c \geq a+\sqrt{3} b$. Suppose it would be $a<\sqrt{3} b$. Then the left hand side of $(2.5)$ is a quadratic polynomial in $c$ with negative leading coefficient, and with zeros $\left(a^{2}+b^{2}\right) /(a-\sqrt{3} b)$ and $\left(a^{2}+b^{2}\right) /(a+\sqrt{3} b)$. Thus, on account of $c \geq 0$, inequality $(2.5)$ is equivalent to $c \leq\left(a^{2}+b^{2}\right) /(a+\sqrt{3} b)$. Hence we obtain the contradiction

$$
a+\sqrt{3} b \leq c \leq \frac{a^{2}+b^{2}}{a+\sqrt{3} b}
$$

and therefore, $a \geq \sqrt{3} b$. Thus we have shown the inclusion

$$
\mathcal{R}(3, E) \subseteq\left\{-a+b \mathrm{i} \in \mathbb{C}^{+}: \sqrt{3} b \leq a\right\} \text { for any } E \in \mathcal{K}_{0}^{n} .
$$


Next we study the boundary, i.e., we suppose that we have $\sqrt{3} b=a$. In this case, (2.4) and (2.5) reduce to $c(c-2 a) \geq 0$ and $c \leq(2 / 3) a$, respectively, because $a>0$. Since $c \geq 0$ we must have $c=0$ and thus $\mathrm{W}_{0}(K ; E)=0$. Then, in view of $\mathrm{W}_{1}(K ; E)>0$ this shows that $\operatorname{dim} K=2$. Moreover, by (2.3) we find $\mathrm{W}_{2}(K ; E)=(2 / 3) a \mathrm{~W}_{3}(K ; E)$ and $\mathrm{W}_{1}(K ; E)=(4 / 9) a^{2} \mathrm{~W}_{3}(K ; E)$, and so

$$
\mathrm{W}_{2}(K ; E)^{2}-\mathrm{W}_{1}(K ; E) \mathrm{W}_{3}(K ; E)=0 .
$$

Now it is known (see [2]) that the above equality holds if and only if $E$ is a cap-body of the 2-dimensional set $K$. Thus if $E \in \mathcal{K}_{0}^{3}$ is not a cap-body of a planar set we have the inclusion $\mathcal{R}(3, E) \subseteq\left\{-a+b \mathrm{i} \in \mathbb{C}^{+}: \sqrt{3} b<a\right\} \cup\{0\}$.

In order to conclude the proof of the theorem we have to show that for any $-a+b \mathrm{i} \in \mathbb{C}^{+}$, with $a>\sqrt{3} b$ or $a=\sqrt{3} b$, depending on the gauge body $E \in \mathcal{K}_{0}^{n}$, there exists $K \in \mathcal{K}^{n}$ such that $f_{K ; E}(-a+b \mathrm{i})=0$. We have to distinguish three cases. If $b=0$ then we just have to consider $K=a E$ for $a>0$ and any planar convex body for $a=0$.

So let $b>0$ and $a>\sqrt{3} b$. Here we take a suitable dilate of a cap-body of $E$. Indeed the complex roots of the Steiner polynomials of all possible cap-bodies of $E$ (see Remark 3.1 when $k, n=3$ ) determine a continuous parametrized curve

$$
\left(-\frac{t+2}{2\left(t^{2}+t+1\right)}, \frac{\sqrt{3} t}{2\left(t^{2}+t+1\right)}\right), \text { for } t \in(0,1)
$$

with limit points $(-1,0),(-1 / 2, \sqrt{3} / 6)$ when $t$ tends to 0 and 1 , respectively (see Figure 2). By Lemma 2.1 i) the complex number $-a+b$ i will be the root of the Steiner polynomial of some dilate of a cap-body of $E$.

Finally, let $b>0$ and $a=\sqrt{3} b$. Here $E \in \mathcal{K}_{0}^{n}$ has to be a cap-body of a planar convex body $K$, and then a dilate of $K$ itself will give the solution. In fact, since $E$ is a cap-body of $K$ then $\operatorname{vol}(E)=\mathrm{W}_{0}(E ; K)=\mathrm{W}_{1}(E ; K)=$ $\mathrm{W}_{2}(E ; K)$ ([19, p. 368, proof of Theorem 6.6.16]), and so noticing that $\mathrm{W}_{0}(K ; E)=0$ we get

$$
\begin{aligned}
f_{K ; E}(z) & =z \sum_{i=1}^{3}\left(\begin{array}{l}
3 \\
i
\end{array}\right) \mathrm{W}_{i}(K ; E) z^{i-1}=z \sum_{i=1}^{3}\left(\begin{array}{l}
3 \\
i
\end{array}\right) \mathrm{W}_{3-i}(K ; E) z^{i-1} \\
& =z \operatorname{vol}(E)\left(3+3 z+z^{2}\right)
\end{aligned}
$$

with non-zero roots $-3 / 2 \pm \sqrt{3} / 2$ i. Lemma 2.1 i) concludes the proof.

Just computing the roots of the Steiner polynomial of 4-dimensional capbodies (see again Remark 3.1) we see that $\mathcal{R}(3, E) \subset \mathcal{R}(4, E)$ strictly.

\section{Characterizing CONvex bodies by properties of the roots}

This section is devoted to characterize (families of) convex bodies by means of properties of the roots. Here we will need the inequalities

$$
\mathrm{W}_{i}(K ; E) \geq \mathrm{r}(K ; E) \mathrm{W}_{i+1}(K ; E), \quad i \in\{0, \ldots, n-1\},
$$


which are a direct consequence of the monotonicity of the mixed volumes (cf. e.g. [19, p. 277]), since, up to translations, $\mathrm{r}(K ; E) E \subseteq K$.

I. $p$-tangential bodies. A convex body $K \in \mathcal{K}^{n}$ containing $E \in \mathcal{K}_{0}^{n}$ is called a $p$-tangential body of $E, p \in\{0, \ldots, n-1\}$, if each support plane of $K$ that is not a support plane of $E$ contains only $(p-1)$-singular points of $K$. Here a boundary point $x$ of $K$ is said to be an $r$-singular point of $K$ if the dimension of the normal cone in $x$ is at least $n-r$. For further characterizations and properties of $p$-tangential bodies we refer to [19, Section 2.2].

So a 0 -tangential body of $E$ is $E$ itself, 1-tangential bodies are just the cap-bodies (see [19, p. 76]) and each $p$-tangential body of $E$ is also a $q$ tangential body for $p<q \leq n-1$. If $K$ is a $p$-tangential body of $E$ then $\mathrm{r}(K ; E)=1$, and the following result by Favard states a characterization of $n$-dimensional $p$-tangential bodies in terms of the quermassintegrals:

Theorem 3.1 (Favard [5], [19, p. 367]). Let $K, E \in \mathcal{K}_{0}^{n}, E \subseteq K$, and let $p \in\{0, \ldots, n-1\}$. Then $K$ is a p-tangential body of $E$ if and only if

$$
\mathrm{W}_{0}(K ; E)=\mathrm{W}_{1}(K ; E)=\cdots=\mathrm{W}_{n-p}(K ; E) .
$$

This property may be rephrased in terms of the roots as follows where we exclude the trivial case of 0 -tangential bodies.

Proposition 3.1. Let $K, E \in \mathcal{K}_{0}^{n}, E \subset K$, let $\gamma_{1}, \ldots, \gamma_{n}$ be the roots of $f_{K ; E}(z)$ and let $p \in\{1, \ldots, n-1\}$. Then $K$ is a $p$-tangential body of $E$ if and only if there exist constants $\alpha_{j}, j=1, \ldots, p$, with $0<\alpha_{p}<1$ such that the $\gamma_{i}$ 's are the roots of the polynomial $g_{\alpha, p}(z)=(z+1)^{n}-\sum_{k=0}^{p-1}\left(\begin{array}{l}n \\ k\end{array}\right) \alpha_{p-k} z^{n-k}$.

Proof. If $K \in \mathcal{K}_{0}^{n}$ is a $p$-tangential body of $E$, Theorem 3.1 asserts that $\mathrm{W}_{0}(K ; E)=\mathrm{W}_{i}(K ; E)$, for all $i=1, \ldots, n-p$, and so we may write

$$
\begin{aligned}
f_{K ; E}(z) & =\mathrm{W}_{0}(K ; E)\left[\sum_{i=0}^{n-p}\left(\begin{array}{c}
n \\
i
\end{array}\right) z^{i}+\sum_{i=n-p+1}^{n}\left(\begin{array}{c}
n \\
i
\end{array}\right) \frac{\mathrm{W}_{i}(K ; E)}{\mathrm{W}_{0}(K ; E)} z^{i}\right] \\
& =\mathrm{W}_{0}(K ; E)\left[(z+1)^{n}-\sum_{k=0}^{p-1}\left(\begin{array}{l}
n \\
k
\end{array}\right)\left(1-\frac{\mathrm{W}_{n-k}(K ; E)}{\mathrm{W}_{0}(K ; E)}\right) z^{n-k}\right] .
\end{aligned}
$$

Thus, setting $\alpha_{p-k}=1-\mathrm{W}_{n-k}(K ; E) / \mathrm{W}_{0}(K ; E), k=0, \ldots, p-1$, all $\gamma_{i}$ 's are roots of the polynomial $g_{\alpha, p}(z)$. Since $\alpha_{p}=1-\operatorname{vol}(E) / \operatorname{vol}(K)$ we have by our assumption $E \subset K$ that $0<\alpha_{p}<1$.

Conversely, we now assume that there exist $p$ constants $\alpha_{j}, j=1, \ldots, p$, $0<\alpha_{p}<1$, such that the $\gamma_{i}$ 's are the roots of $g_{\alpha, p}(z)$. Since $\alpha_{p}<1$ the polynomial $g_{\alpha, p}(z)$ is of degree $n$. Hence the $\gamma_{i}$ 's are roots of the polynomial

$$
(z+1)^{n}-\sum_{k=0}^{p-1}\left(\begin{array}{l}
n \\
k
\end{array}\right) \alpha_{p-k} z^{n-k}=\left[\sum_{i=0}^{n-p}\left(\begin{array}{l}
n \\
i
\end{array}\right) z^{i}+\sum_{i=n-p+1}^{n}\left(\begin{array}{l}
n \\
i
\end{array}\right)\left(1-\alpha_{p+i-n}\right) z^{i}\right]
$$


as well as of $f_{K ; E}(z)=\sum_{i=0}^{n}\left(\begin{array}{c}n \\ i\end{array}\right) \mathrm{W}_{i}(K ; E) z^{i}$. So both polynomials have the same coefficients up to a constant $c$, say. Since $\alpha_{p}<1$ we have $c>0$ and by comparing the coefficients we get

$$
\mathrm{W}_{0}(K ; E)=\mathrm{W}_{1}(K ; E)=\cdots=\mathrm{W}_{n-p}(K ; E)=c .
$$

Theorem 3.1 now ensures that $K$ is a $p$-tangential body of $E$.

We note that the constants $\alpha_{j}$ 's in the theorem above actually satisfy $0<\alpha_{1} \leq \cdots \leq \alpha_{p}<1$, which follows directly from (3.1) since $\operatorname{r}(K ; E)=1$.

In the particular case of cap-bodies (1-tangential bodies) the characterization is more explicit.

Corollary 3.1. Let $K, E \in \mathcal{K}_{0}^{n}, E \subset K$, and let $\gamma_{1}, \ldots, \gamma_{n}$ be the roots of $f_{K ; E}(z)$. Then $K$ is a cap-body of $E$ if and only if there exists $\alpha \in(0,1)$ such that $(1 / \alpha)^{1 / n}\left(1+1 / \gamma_{i}\right), i=1, \ldots, n$, are the $n$ roots of unity.

Proof. Proposition 3.1 states that $K$ is a 1-tangential body of $E$ if and only if there exists $\alpha \in(0,1)$ such that $\gamma_{k}, k=1, \ldots, n$, satisfy the equation $(z+1)^{n}=\alpha z^{n}$. Since $\mathrm{W}_{0}(K, E)>0$ no root of $f_{K ; E}(z)$ can be zero and so we have equivalently $(1+1 / z)^{n}=\alpha$. Therefore, $K$ is a 1-tangential body of $E$ if and only if

$$
1+\frac{1}{\gamma_{k}}=\alpha^{1 / n} \mathrm{e}^{\frac{2 \pi(k-1)}{n} \mathrm{i}}, \quad \text { for } k=1, \ldots, n
$$

and some $\alpha \in(0,1)$.

Remark 3.1. Obviously, with (3.2) we can explicitly determine the roots of the Steiner polynomial of a cap-body $K$ of $E$, namely

$$
\gamma_{k}=\frac{-1+\beta \cos \frac{2 \pi(k-1)}{n}-\beta \sin \frac{2 \pi(k-1)}{n} \mathrm{i}}{1+\beta\left(\beta-2 \cos \frac{2 \pi(k-1)}{n}\right)}, \quad k=1, \ldots, n,
$$

with $\beta=\alpha^{1 / n}=(1-\operatorname{vol}(E) / \operatorname{vol}(K))^{1 / n}$. Figure 2) shows in a thicker line all roots in $\mathcal{R}\left(3, B_{3}\right)$ of Steiner polynomials of cap-bodies of $B_{3}$ (cf. Figure 1).

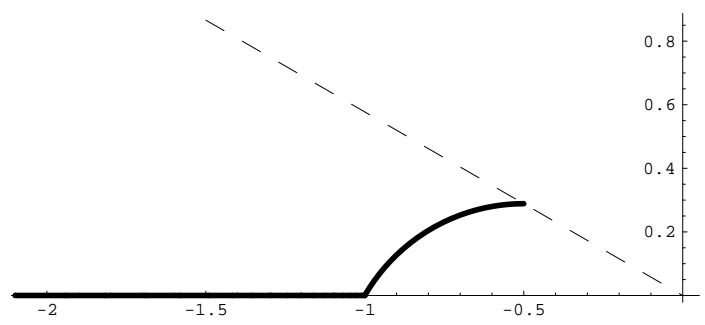

Figure 2. Complex numbers $z \in \mathbb{C}^{+}$which are roots of $f_{K ; B_{3}}(z)$ for any cap-body $K$ of $B_{3}$. 
II. On constant width sets. Next we deal with the family of constant width sets, for which we get a symmetry condition on the roots of their Steiner polynomials. A convex body $K$ is called a constant width set if it has the same breath (say b) in any direction, i.e., the distance between any two parallel supporting hyperplanes is b.

In order to establish this symmetry condition we need the following general result on polynomials.

Lemma 3.1. Let $f(z)=a_{0}+a_{1} z+\cdots+a_{n} z^{n}$ be a real polynomial with $a_{n} \neq 0$ and let $b \in \mathbb{R}$. Then

$$
a_{n-k}=\sum_{i=0}^{k}(-1)^{i}\left(\begin{array}{c}
n-i \\
n-k
\end{array}\right) b^{k-i} a_{n-i}
$$

for $k=0,1, \ldots, n$, if and only if all the roots of $f(z)$ are symmetric with respect to $-b / 2$, i.e., $\gamma$ is a root if and only if $-b-\gamma$ is a root.

Proof. The polynomial $f(-b-z)$ is given by

$$
\begin{aligned}
f(-b-z) & =\sum_{k=0}^{n} a_{k}(-b-z)^{k}=\sum_{k=0}^{n} a_{k}(-1)^{k}\left(\sum_{i=0}^{k}\left(\begin{array}{c}
k \\
i
\end{array}\right) b^{k-i} z^{i}\right) \\
& =\sum_{k=0}^{n}\left(\sum_{m=k}^{n} a_{m}(-1)^{m}\left(\begin{array}{c}
m \\
k
\end{array}\right) b^{m-k}\right) z^{k} \\
& =\sum_{k=0}^{n}\left(\sum_{l=0}^{n-k} a_{n-l}(-1)^{n-l}\left(\begin{array}{c}
n-l \\
k
\end{array}\right) b^{n-k-l}\right) z^{k} \\
& =(-1)^{n} \sum_{k=0}^{n}\left(\sum_{l=0}^{n-k} a_{n-l}(-1)^{l}\left(\begin{array}{c}
n-l \\
k
\end{array}\right) b^{n-k-l}\right) z^{k} .
\end{aligned}
$$

The leading coefficient is $(-1)^{n} a_{n}$ and thus the two polynomials $f(z)$ and $f(-b-z)$ have the same roots if and only if $f(z)=(-1)^{n} f(-b-z)$, i.e., if and only if the relations (3.4) hold for all $k=0,1, \ldots, n$.

As a direct consequence we get the following statement for Steiner polynomials.

Theorem 3.2. Let $\mathrm{b}>0$. A convex body $K \in \mathcal{K}^{n}$ verifies the relations

$$
\mathrm{W}_{n-k}(K ; E)=\sum_{i=0}^{k}(-1)^{i}\left(\begin{array}{l}
k \\
i
\end{array}\right) \mathrm{b}^{k-i} \mathrm{~W}_{n-i}(K ; E)
$$

for $k=0,1, \ldots, n$, if and only if all the roots of its Steiner polynomial $f_{K ; E}(z)$ are symmetric with respect to $-\mathrm{b} / 2$.

Proof. Since

$$
\left(\begin{array}{l}
n \\
k
\end{array}\right)\left(\begin{array}{l}
k \\
i
\end{array}\right)=\left(\begin{array}{l}
n \\
i
\end{array}\right)\left(\begin{array}{c}
n-i \\
n-k
\end{array}\right) \quad \text { for } i \leq k,
$$

the result follows immediately from Lemma 3.1 . 
Observe that the equations (3.6) are not independent, since they are equivalent to the $(n+1) / 2$ relations obtained for only odd values of $k$.

Notice also that symmetry with respect to $\mathrm{b} / 2 \geq 0$ in Theorem 3.2 is not possible. Indeed, for $\mathrm{b}=0$ we would get that all odd quermassintegrals have to vanish which is not possible, and in the case $\mathrm{b}>0$ it would imply that $\mathrm{W}_{j}(K ; E)=0$ for all $j=1, \ldots, n$, again not possible.

When $E=B_{n}$, it is known (see [4]) that bodies of constant width fulfill (3.6), which shows Proposition 1.2 .

In particular, if $n=3$ the roots of the Steiner polynomial of the constant width set $K$ are

$$
\gamma_{1}=-\frac{\mathrm{b}}{2}, \quad \gamma_{2}, \gamma_{3}=-\frac{\mathrm{b}}{2} \pm \frac{\sqrt{3}}{2} \sqrt{\mathrm{b}^{2}-\frac{3 \mathrm{~W}_{1}(K)}{\pi}},
$$

which are real, since $\pi \mathrm{b}^{2} \geq 3 \mathrm{~W}_{1}(K)$ (cf. (2.1); see also [8, Example 3]).

Notice that if (3.6) holds and the dimension is odd then $-\mathrm{b} / 2$ is a root of $f_{K ; E}(z)$, whereas if $n$ is even and $-\mathrm{b} / 2$ is a root of $f_{K ; E}(z)$, then it has to be at least a double root.

III. Balls and sausages. Finally we consider for $K$ balls (more generally the gauge body $E$ ) and sausages. The gauge body $E$ can be seen both as a very special tangential body (it is a 0 -tangential body of $E$ ) and as a very particular case of a body satisfying (3.6). It can be also characterized in a more general way:

Proposition 3.2. Let $K \in \mathcal{K}^{n}$, let $\gamma_{i}, i=1, \ldots, n$, be the roots of $f_{K ; E}(z)$, and let $a>0$. Then $\left|\operatorname{Re}\left(\gamma_{i}\right)\right|=a>0$ for all $i=1, \ldots, n$ if and only if $K=a E$ (up to translations).

Proof. If $K=a E$, then $f_{K ; E}(z)=\mathrm{W}_{n}(K ; E)(z+a)^{n}$, and hence it has an $n$-fold real root $\gamma=-a$. So let us assume $\left|\operatorname{Re}\left(\gamma_{i}\right)\right|=a$ for $i=1, \ldots, n$. From $f_{K ; E}(z)=\mathrm{W}_{n}(K ; E) \prod_{i=1}^{n}\left(z-\gamma_{i}\right)$ we get the relations

$$
\begin{aligned}
&-m a=\sum_{i=1}^{n} \operatorname{Re}\left(\gamma_{i}\right)= \sum_{i=1}^{n} \gamma_{i}=-n \frac{\mathrm{W}_{n-1}(K ; E)}{\mathrm{W}_{n}(K ; E)}, \\
& \prod_{i=1}^{n} \gamma_{i}=(-1)^{n} \frac{\mathrm{W}_{0}(K ; E)}{\mathrm{W}_{n}(K ; E)},
\end{aligned}
$$

for some $1 \leq m \leq n$. We note that we can exclude the case $m=0$, because $a>0$ implies $K \in \mathcal{K}_{0}^{n}$ and thus $\mathrm{W}_{n-1}(K ; E) \neq 0$. Hence

$$
\frac{\mathrm{W}_{0}(K ; E)}{\mathrm{W}_{n}(K ; E)}=\prod_{i=1}^{n}\left|\gamma_{i}\right| \geq a^{n}=\left(\frac{n}{m} \frac{\mathrm{W}_{n-1}(K ; E)}{\mathrm{W}_{n}(K ; E)}\right)^{n} \geq \frac{\mathrm{W}_{n-1}(K ; E)^{n}}{\mathrm{~W}_{n}(K ; E)^{n}},
$$

i.e., $\mathrm{W}_{n}(K ; E)^{n-1} \mathrm{~W}_{0}(K ; E) \geq \mathrm{W}_{n-1}(K ; E)^{n}$. However Minkowski's first inequality states that $\mathrm{W}_{n}(K ; E)^{n-1} \mathrm{~W}_{0}(K ; E) \leq \mathrm{W}_{n-1}(K ; E)^{n}$, where equality holds for $K, E \in \mathcal{K}_{0}^{n}$ if and only if $K$ and $E$ are homothetic (see e.g. [19, p. 317]). Thus there exist $\alpha>0$ and $t \in \mathbb{R}^{n}$ such that $K=\alpha E+t$. From 
$a^{n}=\mathrm{W}_{0}(K ; E) / \mathrm{W}_{n}(K ; E)$ we get $\alpha=a$, and, in particular, $m=n$ and $\gamma_{i}=-a$ for all $i=1, \ldots, n$.

Theorem 1.3 will be a direct consequence of the following result for which we denote by $f_{K ; B_{n}}^{(n-1)}(z)$ the $(n-1)$-st derivative of $f_{K ; B_{n}}(z)$.

Theorem 3.3. Let $K \in \mathcal{K}^{n}$. Then $-\mathrm{r}(K)$ is root of $f_{K ; B_{n}}^{(n-1)}(z)$ and all its 2-dimensional projections onto any 2-dimensional linear subspace have inradius $\mathrm{r}(K)$ if and only $K$ is a sausage with inradius $\mathrm{r}(K)$.

Proof of Theorem 3.3. First we need some additional notation. The set of all $k$-dimensional linear subspaces of $\mathbb{R}^{n}$ will be denoted by $\mathcal{L}_{k}^{n}$, and for $L \in \mathcal{L}_{k}^{n}$ and $K \in \mathcal{K}^{n}, K \mid L$ denotes the orthogonal projection of $K$ onto $L$. Finally, $\mathrm{W}_{i}^{(k)}$ denotes the $i$-th quermassintegral computed in a $k$-dimensional affine subspace.

The quermassintegrals of a sausage $S=L+\mathrm{r} B_{n}$, where $L$ is a line segment with length $\ell$, are given by

$$
\mathrm{W}_{k}(S)=\mathrm{r}^{n-k-1}\left[\frac{n-k}{n} \operatorname{vol}_{n-1}\left(B_{n-1}\right) \ell+\operatorname{vol}\left(B_{n}\right) \mathrm{r}\right] .
$$

Here $\operatorname{vol}_{j}(M)$ denotes the $j$-dimensional volume of $M \subset \mathbb{R}^{j}$. Thus it can be easily checked that $-\mathrm{r}=-\mathrm{r}(S)$ is an $(n-1)$-fold root of $f_{S ; B_{n}}(z)$ and so a root of its $(n-1)$-st derivative.

So we assume that $-\mathrm{r}=-\mathrm{r}(K)$ is root of $f_{K ; B_{n}}^{(n-1)}(z)$, i.e.,

$$
\mathrm{W}_{n-2}(K)-2 \mathrm{rW}_{n-1}(K)+\mathrm{r}^{2} \mathrm{~W}_{n}(K)=0,
$$

and let $\mathrm{r}\left(K \mid L ; B_{n} \cap L\right)=\mathrm{r}(K)$ for all projections onto 2-dimensional planes $L \in \mathcal{L}_{2}^{n}$. Let $k \in\{1, \ldots, n\}$. Kubota's integral recursion formula states that for any $i=0, \ldots, k$, the $(n-k+i)$-th quermassintegral $\mathrm{W}_{n-k+i}(K)$ can be expressed as

$$
\mathrm{W}_{n-k+i}(K)=\frac{\operatorname{vol}\left(B_{n}\right)}{\operatorname{vol}_{k}\left(B_{k}\right)} \int_{\mathcal{L}_{k}^{n}} \mathrm{~W}_{i}^{(k)}(K \mid L) d \sigma(L),
$$

where $\sigma(L)$ is the Haar measure on the set $\mathcal{L}_{k}^{n}$ such that $\sigma\left(\mathcal{L}_{k}^{n}\right)=1$ (see e.g. $[19$, p. $295,(5.3 .27)])$.

Let $k=2$ and $L \in \mathcal{L}_{2}^{n}$. The Steiner polynomial of $K \mid L$ as a 2-dimensional set is given by $f_{K \mid L ; B_{2}}(z)=\sum_{i=0}^{2}\left(\begin{array}{c}2 \\ i\end{array}\right) \mathrm{W}_{i}^{(2)}(K \mid L) z^{i}$. Applying (3.9) we get

$$
\begin{aligned}
\int_{\mathcal{L}_{2}^{n}} f_{K \mid L ; B_{2}}(-\mathrm{r}) d \sigma(L)= & \int_{\mathcal{L}_{2}^{n}} \mathrm{~W}_{0}^{(2)}(K \mid L) d \sigma(L)-2 \mathrm{r} \int_{\mathcal{L}_{2}^{n}} \mathrm{~W}_{1}^{(2)}(K \mid L) d \sigma(L) \\
& +\mathrm{r}^{2} \int_{\mathcal{L}_{2}^{n}} \mathrm{~W}_{2}^{(2)}(K \mid L) d \sigma(L) \\
= & \frac{\operatorname{vol}_{2}\left(B_{2}\right)}{\operatorname{vol}\left(B_{n}\right)}\left[\mathrm{W}_{n-2}(K)-2 \mathrm{rW}_{n-1}(K)+\mathrm{r}^{2} \mathrm{~W}_{n}(K)\right]=0
\end{aligned}
$$


by (3.8). Since $\mathrm{r}=\mathrm{r}\left(K \mid L ; B_{n} \cap L\right)$ for all $L \in \mathcal{L}_{2}^{n}$, Bonnesen's inequality (cf. (1.4)) states that

$$
f_{K \mid L ; B_{2}}(-\mathrm{r})=\mathrm{W}_{0}^{(2)}(K \mid L)-2 \mathrm{~W}_{1}^{(2)}(K \mid L) \mathrm{r}+\mathrm{W}_{2}^{(2)}(K \mid L) \mathrm{r}^{2} \leq 0,
$$

with equality if and only if $K \mid L$ is a 2-dimensional sausage with inradius r. Thus, from

$$
\int_{\mathcal{L}_{2}^{n}} f_{K \mid L ; B_{2}}(-\mathrm{r}) d \sigma(L)=0
$$

and (3.10) we conclude $f_{K \mid L ; B_{2}}(-\mathrm{r})=0$ for any $L \in \mathcal{L}_{2}^{n}$ which implies that $K \mid L$ is a 2-dimensional sausage with inradius $\mathrm{r}$ for any $L \in \mathcal{L}_{2}^{n}$. By [19, Lemma 3.2.6] this is equivalent to $K$ being an $n$-dimensional sausage with inradius $\mathrm{r}$.

\section{REFERENCES}

[1] W. Blaschke, Vorlesungen über Integralgeometrie, Deutscher Verlag der Wissenschaften, Berlin, 1995, 3rd ed. (1st ed.: 1949).

[2] G. Bol, Beweis einer Vermutung von H. Minkowski, Abh. Math. Sem. Hansischen Univ. 15 (1943), 37-56.

[3] T. Bonnesen, Les problèmes des isopérimètres et des isépiphanes, Collection de monographies sur la théorie des fonctions, Gauthier-Villars, Paris 1929.

[4] A. Dinghas, Verallgemeinerung eines Blaschkeschen Satzes über konvexe Körper konstanter Breite, Rev. Math. Union Interbalkan. 3 (1940), 17-20.

[5] J. Favard, Sur les corps convexes, J. Math. Pures Appl. 12 (9) (1933), 219-282.

[6] P. M. Gruber, Convex and Discrete Geometry. Springer, Berlin Heidelberg, 2007.

[7] M. Henk, M. A. Hernández Cifre, Notes on the roots of Steiner polynomials, Rev. Mat. Iberoamericana 24 (2) (2008), 631-644.

[8] M. A. Hernández Cifre, E. Saorín, On the roots of the Steiner polynomial of a 3dimensional convex body, Adv. Geom. 7 (2007), 275-294.

[9] M. A. Hernández Cifre, E. Saorín, Some geometric properties of the roots of the Steiner polynomial, Rend. Circ. Mat. Palermo 77 (II) (2006), 319-332.

[10] M. A. Hernández Cifre, E. Saorín, How to make quermassintegrals differentiable: solving a problem by Hadwiger, Submitted.

[11] M. Jetter, Bounds on the roots of the Steiner polynomial, to appear in Adv. Geom.

[12] O. M. Katkova, A. M. Vishnyakova, A sufficient condition for a polynomial to be stable, J. Math. Anal. Appl. 347 (1) (2008), 81-89.

[13] V. Katsnelson, On H. Weyl and J. Steiner polynomials, Complex Anal. Oper. Theory 3 (1) (2009), 147-220.

[14] M. Marden, Geometry of polynomials, Mathematical Surveys, No. 3, American Mathematical Society, Providence, R.I., 1966, 2nd ed.

[15] G. Matheron, La formule de Steiner pour les érosions, J. Appl. Prob. 15 (1978), 126-135.

[16] Y. Y. Nie, X. K. Xie, New criteria for polynomial stability, IMA J. Math. Control Inform. 4 (1) (1987), 1-12.

[17] J. R. Sangwine-Yager, Bonnesen-style inequalities for Minkowski relative geometry. Trans. Amer. Math. Soc. 307 (1) (1988), 373-382.

[18] J. R. Sangwine-Yager, Mixed volumes. In: Handbook of Convex Geometry (P. M. Gruber and J. M. Wills eds.), North-Holland, Amsterdam, 1993, 43-71.

[19] R. Schneider, Convex Bodies: The Brunn-Minkowski Theory, Cambridge University Press, Cambridge, 1993. 
[20] J. Steiner, Über parallele Flächen, Monatsber. Preuss. Akad. Wiss. (1840), 114-118, [Ges. Werke, Vol II (Reimer, Berlin, 1882) 245-308].

[21] B. Teissier, Bonnesen-type inequalities in algebraic geometry I. Introduction to the problem. Seminar on Differential Geometry, Princeton Univ. Press, Princeton, N. J., 1982, 85-105.

Fakultät für Mathematik, Otto-von-Guericke Universität Magdeburg, UniVersitätsplatz 2, D-39106-Magdeburg, Germany

E-mail address: martin.henk@ovgu.de

Departamento de Matemáticas, Universidad de Murcia, Campus de EspinarDO, 30100-MurCIA, SPAIN

E-mail address: mhcifre@um.es 